Revista Acta Ambiental Catarinense - Unochapecó.

Edição: v. 18 n. 1 (2021): Temática Livre - jan. / dez.

Editores: Dr. Dr. Junir Antonio Lutinski, Dr. Dr. Renan de Souza Rezende, Mrs. Edpool Rocha Silva, Mrs. João Carlos Valentim Veiga Junior, Dr. Juliano Luiz Fossá.

MINERAL AND ORGANIC FERTILIZERS INFLUENCE THE ATTRACTION OF Avena strigosa ROOTS AND THE BEHAVIOR OF Folsomia candida

Autores: Daniel Augusto Barreta, Suélen Serafini, Luís Carlos luñes Oliveira Filho, Antonio Waldimir Leopoldino da Silva, Alexandre Tadeu Paulino, Dilmar Baretta

ANÁLISE DO POTENCIAL BIOCATALÍTICO DE LIPASE DE CANDIDA RUGOSA IMOBILIZADA EM DIFERENTES SUPORTES: POTENCIAL BIOCATALÍTICO DE LIPASE DE CANDIDA RUGOSA

Autores: Adriano da Silva, Rafael José Resende, Tamires Cristina Costa, Bárbara Viviane Oliveira de Sousa, Anderson Kenedy Santos, Braz de Souza Marotti, Saulo Luís da Silva, Adriano Cancelier, Daniel Bonoto Gonçalves

COMPOSIÇÃO DA ICTIOFAUNA DO CÓRREGO BEBEDOURO (FRUTAL, MG) E SUA RELAÇÃO COM FATORES AMBIENTAIS: ICTIOFAUNA E SUA RELAÇÃO COM FATORES AMBIENTAIS

Autores: Júlio César dos Santos Lima, Renata Cassemiro Biagioni, Cristiano Pereira da Cunha, Vitor Loreno de Almeida Cerqueira, Ariane Almeida Vaz, Adriane Almeida Vaz, Cleber Coelho Machado, Sofia Luiza Brito, Tânia Aparecida da Silva Brito, Welber Senteio Smith

PERFIL DA PRODUÇÃO AGRÍCOLA TEMPORÁRIA NA REGIÃO DE SÃO CARLOS, SP: UM BALANÇO DOS ÚLTIMOS 12 ANOS

Autores: Bruno Salata Lima, Risely Ferraz-Almeida

ORIGEM FITOGEOGRÁFICA, ESTRUTURA FILOGENÉTICA E SÍNDROMES DE DISPERSÃO DE ESPÉCIES ARBÓREAS EM FLORESTA OMBRÓFILA MISTA NO PLANALTO CATARINENSE: Ecologia e Fitogeografia em Área de Floresta Ombrófila Mista

Autores: Larissa Cardoso Küster, Pedro Higuchi, Ana Carolina da Silva, Roni Djeison Ansolin, Marco Antônio Bento, Fernando Buzzi Júnior, Tiago de Souza Ferreira

LEVANTAMENTO DA FERTILIDADE DO SOLO DE PROPRIEDADES RURAIS DO MUNÍCIPIO DE CHAPECÓ - SC: UM ESTUDO DE CASO: Levantamento da fertilidade do solo

Autores: Alana Maria Polesso, Evandro Spagnollo, Carolina Riviera Duarte Maluche Baretta

\title{
Os EXTRATOS VEGETAIS AQUOSOS PARA O TRATAMENTO DE SEMENTES DE CÁRTAMO
}

Tratamento de sementes alternativos

Autores: Janine Farias Menegaes, Ubirajara Russi Nunes, Marlove Fátima Brião Muniz, Rogério Antonio Bellé, Priscila Barbieri Zini

GROWTH RATES AND PREY-HANDLING BEHAVIOR OF HATCHLING HELICOPS ANGULATUS (LINNAEUS, 1758) (SERPENTES; HYDROPSINI) IN THE ATLANTIC FOREST OF NORTHEASTERN BRAZIL: Growth rates and prey-handling behavior of hatchling Helicops angulatus Autores: Jéssica Monique da Silva Amaral, Vanessa do Nascimento Barbosa, Ivan Lívio Rocha Sampaio, Mayara da Silva Ribeiro de Morais, Frederico Gustavo Rodrigues França. 
Acta Ambiental Catarinense - Unochapecó

oper @access

TRATAMENTO AERÓBIO DE ESGOTO EMPREGANDO A TÉCNICA DE BIOAUMENTAÇÃO: Tratamento aeróbio de esgoto por bioaumentação

Autores: Guilherme Pereira de Matos, Jéssica Talita Zagonel

MUDANÇAS NO CLIMA E A DISTRIBUIÇÃO POTENCIAL FUTURA DE Mimosa scabrella BENTH Influência das mudanças climáticas na bracatinga

Autores: Mariéle Alves Ferrer da Silva, Pedro Higuchi, Ana Carolina da Silva

REGISTRO DE FRUGIVORIA EM FURÃO-PEQUENO (GALICTIS CUJA) NO BRASIL: Frugivoria por Galictis cuja

Autores: Artur Schmitt, Mario Arthur Favretto

EFEITO DA PULVERIZAÇÃO FOLIAR DE NUTRIENTES NO MANEJO FITOSSANITÁRIO E NA PRODUTIVIDADE DE CEBOLA: Manejo fitossanitário e produtividade de cebola

Autores: Paulo Antonio de Souza Gonçalves, Edivânio Rodrigues de Araújo, Claudinei Kurtz, Leandro Delalibera Geremias, Cristiano Mora

EFEITO DAS CINZAS DO BAGAÇO DE CANA-DE-AÇÚCAR E BAMBU SOBRE QUALIDADE DE FIBROCIMENTOS EXTRUDADOS

Autores: Mateus Alexandre da Silva, Alan Pereira Vilela, Tony Matheus Carvalho Eugênio, Danilo Wisky Silva, Rafael Farinassi Mendes

OBTENÇÃO E CARACTERIZAÇÃO DE COMPÓSITOS POLIMÉRICOS BIODEGRADÁVEIS PRODUZIDOS COM RESÍDUOS AGROENERGÉTICOS (BAGAÇO DA CANA-DE-AÇÚCAR, AMIDO DE MILHO E GLICEROL)

Autores: Roberta Samara Barros Nunes, Alexandre Albuquerque do Nascimento, Juan Carlos Valdés Serra

POTENCIAL FITOSSANITÁRIO DE SEMENTES DE CÁRTAMO ARMAZENADAS EM DIFERENTES CONDIÇÕES DE CONSERVAÇÃO E PERÍODOS

Autores: Janine Farias Menegaes, Rogério Antônio Bellé, Ubirajara Russi Nunes

BIOFERTILIZANTE DE ESTERCO BOVINO NA INDUÇÃO DE MECANISMOS DE DEFESA À MELOIDOGYNE INCOGNITA NA CULTURA DA SOJA

Autores: Jessica Brasau da Silva, Juliana Santos Batista Oliveira, Camila Jorge B. Ferreira, Antônio Jussiê Da Silva Solino, Kátia R. F. Schwan-Estrada

PERFORMANCE OF ENTOMOPATHOGENIC NEMATODES ON NEOLEUCINODES ELEGANTALIS (GUENÉE) (LEPIDOPTERA: CRAMBIDAE)

Autores: Dirceu Pratissoli, ALIXELHE PACHECO DAMASCENA, Débora Melo Ferreira Fragoso, José Romário de Carvalho, Amanda Carlos Túler, Luana Viana Faria, Luis Moreira de Araujo Junior

GERMINAÇÃO DE SEMENTES DE ARAUCÁRIA SUBMETIDAS A DIFERENTES CONDIÇÕES HÍDRICAS DE ARMAZENAMENTO

Autores: Daniele Fátima Balhuk, Rogério Antonio Krupek

MICRORGANISMOS NO SEDIMENTO DE MARGENS OPOSTAS DO ESTUÁRIO DO RIO COREAÚ EM CAMOCIM/CE

Autores: Adelaide Sampaio Oliveira, Jardielen Chaves Sousa, Antonio Lucas Rocha Santos, Renatta Santos Oliveira, Dionéia Evangelista Cesar, Edmo Montes Rodrigues 
RECURSOS NATURAIS: CONCEPÇÕES E PRÁTICAS NO AMBIENTE ESCOLAR

Autores: Édson dos Santos Pereira, Rogério Rodrigues Faria, Tatiane do Nascimento Lima

RADIAÇÃO ULTRAVIOLETA COMO ALTERNATIVA TECNOLÓGICA PARA DESINFECÇÃO DE EFLUENTE DE INDÚSTRIA DE PAPEL

Autores: Karoline Mello Millek, Jeanette Beber Souza, Carlos Magno de Sousa Vidal

ANÁLISE DE AGRUPAMENTO BASEADO EM CARACTERÍSTICAS MORFOLÓGICAS DE LEGUMINOSAS DE VÁRZEA COM FINS TAXONÔMICOS

Autores: Rubiene Neto Soares, Ronaldo Oliveira dos Santos, Robson Borges de Lima, Breno Marques da silva e Silva

PROPORÇÕES DE CAMA DE AVIÁRIO NA FORMULAÇÃO DE SUBSTRATO PARA PRODUÇÃO DE MUDAS DE MELANCIA

Autores: Ivan Carlos Chiapinotto, Cristiano Nunes Nesi, Matheus Santin Padilha, Leandro do Prado Wildner, Dirceu Júnior Ferri, Paulo Roberto Ficagna, Carolina Riviera Duarte Maluche Baretta 